\title{
Route Construction for Long Lifetime in VANETs
}

\author{
Nikoletta Sofra, Member, IEEE, Athanasios Gkelias, Member, IEEE, and Kin K. Leung, Fellow, IEEE
}

\begin{abstract}
One of the most distinguishing features of vehicular ad hoc networks (VANETs) is the increased mobility of the nodes. This results in the existence of transient communication links, which degrade the performance of developed protocols. Established routes frequently become invalid, and existing communication flows are interrupted, incurring delay and additional overhead. In this paper, we aim to provide a metric to support the design of networks that can proactively adapt to a constantly changing topology. We present a method that produces a linklifetime-related metric capable of capturing the remaining time for which a link can be used for efficient communication. The metric is intended to be used to optimize route construction with respect to lifetime. We propose a cross-layer approach, which utilizes physical layer information, and formulate the relevant parameter estimation problem. Contrary to existing work, the method does not assume knowledge of the transmission power or the nodes' position and velocity vectors, or adoption of a specific mobility model, whereas the estimates go beyond describing the tendency of link quality. We achieve this by employing a unified model that accurately captures the effect of the radio propagation and the underlying structure of vehicle movement on the temporal dependence of the quality of a wireless mobile link. More specifically, the model takes into account the inherent nonlinearities arising and, most importantly, includes the minimum distance that will be achieved between two vehicles on the course of their movement, which is shown to play a crucial role in the link duration. We present an analytical framework, which quantifies the probability of correctly identifying the longest living link between two given links, based on the estimates. Utilization of the estimates is shown to lead to optimal performance under ideal channel conditions. The proposed scheme outperforms existing affinity-based schemes, achieving to opt for links that last up to $35 \%$ longer under the presence of shadow fading. Finally, we discuss the integration of the proposed estimation method in routing and demonstrate that our estimations can be beneficial, leading to the construction of routes that consistently last longer than routes that have been constructed based on the smoothed signal-to-noise ratio metric.
\end{abstract}

Index Terms-Link lifetime estimation, link residual time (LRT), vehicular ad hoc networks (VANETs), vehicle-to-vehicle communications.

Manuscript received July 22, 2010; revised January 13, 2011 and April 29, 2011; accepted June 13, 2011. Date of publication July 14, 2011; date of current version September 19, 2011. This paper forms part of the MESSAGE project. MESSAGE was a three-year research project that started in October 2006 and was jointly funded by the U.K. Engineering and Physical Sciences Research Council and the U.K. Department for Transport. The project also has the support of 19 nonacademic organizations from public sector transport operations, commercial equipment providers, systems integrators, and technology suppliers. More information is available from the web site www.message-project.org. The views expressed in this paper are those of the authors and do not represent the view of the Department for Transport or any of the nonacademic partners of the MESSAGE project. The review of this paper was coordinated by Dr. F. Bai.

N. Sofra was with Imperial College London, SW7 2AZ London, U.K. She is now with Audi Electronic Venture GmbH, 85080, Gaimersheim, Germany (e-mail: nsofra@ic.ac.uk).

A. Gkelias and K. K. Leung are with the Communications and Signal Processing Group, Electrical and Electronic Engineering Department, Imperial College London, SW7 2AZ London, U.K. (e-mail: a.gkelias@ic.ac.uk; kin.leung@imperial.ac.uk).

Digital Object Identifier 10.1109/TVT.2011.2161787

\section{INTRODUCTION}

$\mathbf{T}$ HE FIELD of vehicular ad hoc networks (VANETs) is constantly drawing research attention due to their wide range of technological applications and the technical challenges they exhibit. Forming a subclass of mobile ad hoc networks (MANETs), VANETs are distinctive from generic MANETs in many ways [1]. The most prominent feature of VANETs is the high mobility of the nodes, which is the underlying cause of a series of VANET-specific attributes requiring the development of applicable solutions. Transient connectivity due to node mobility is an inherent attribute of all mobile networks, which becomes even more evident in the case of vehicular communications. This causes significant problems as communication is disrupted very often, resulting in poor performance. The constantly changing topology has numerous adverse effects on the efficiency of the operations of higher layers on the protocol stack. More specifically, routing design for VANETs is particularly challenging. As the dynamics involved imply that the underlying connectivity capabilities change rapidly, collected routing information becomes stale, and established communication routes become invalid in a short time. The resulting disruption of information flow causes considerable delays, and route reconstruction depletes a significant amount of network resources.

Toward the goal of efficient design of VANETs, the aim of this paper is to develop a method that will allow the estimation of the time for which an existing route will continue to operate satisfactorily, which is defined as the route residual time (RRT), and demonstrate how such a method may be integrated in routing protocols. For the route to continue to be valid for a given period of time, all the individual links comprising the route need to continue to be available. In other words, the lifetime of a route is determined by the lifetime of its "weakest" link. A wireless link on the VANET context will be available until the channel quality deteriorates so much that it drops below a given communication threshold. This reduces the problem of estimating RRT to the estimation of the remaining time for which a link's quality will remain above the specified threshold, which is defined as the link residual time (LRT).

The importance of ploiting the nonrandom behavior of the nodes' mobility patterns to construct long-lived routes and combat frequent communication disconnections was established in [2], where the authors propose the utilization and dissemination of GPS information to calculate the expected connection duration and demonstrate significant performance enhancement when the routing procedure provisionally takes lifetime into account.

Recent research has unveiled route lifetime as an important performance factor for VANETs. If the established routes are short lived, then the route reconstructions will more frequently 
be necessary. In addition, there is no guarantee that the new route will not fail immediately or very shortly after its establishment, causing a series of successive route reconstructions and giving rise to a "ping-pong" behavior. This problem has been underlined in [1], where the authors note that VANETs suffer from a very limited network diameter, as many routes fail before they can be utilized, and in [3], where the authors observed the same behavior in practical implementations of typical MANET protocols. This degrades the performance of developed routing algorithms and implies that there is a significant routing overhead to no avail, introducing further delay in the establishment of the required route and hindering existing communication in the network. The failure of a single radio link is sufficient to render an established route unusable; since one link might be part of multiple routes, several route reconstructions will be necessary at the same time, incurring the corresponding overhead and resulting in extended delivery delay [4]. Specifically for links active in transmission control protocol (TCP) connections, frequent link disconnections cause abnormal TCP behavior [5]. The packet loss will trigger the congestion mechanism of TCP, decreasing the throughput even further.

Our goal is to develop a method that produces a linklifetime-related metric using only physical layer information yet capable of capturing the remaining lifetime of a link in the VANET context accurately enough so that it can be used as a route construction metric. The motivation of this paper lies in the need for a link lifetime metric based on physical layer measurements, which would be suitable for inclusion in cost functions used for route construction. Using physical layer information lifts the assumption of accurate knowledge of the position and velocity vectors of all the nodes and offers the advantage of accommodating for varying transmission power, nondeterministic radio range, and various mobility models. To the best of our knowledge, utilization of physical-layer-related information in VANET routing has been limited to providing criteria that facilitate the exclusion of certain links from the routing decision, ${ }^{1}$ or the initiation of handoffs, rather than developing a metric that can be used as a route construction criterion. In other words, existing link quality metrics have not been demonstrated to optimize route construction with respect to the link lifetime but have rather focused on eliminating links that are expected to have a very short lifetime or on predictors supporting provisional route reconstructions. The proposed routing protocols, which explicitly include route lifetime as a metric for route construction, derive the expected lifetimes via node location and velocity information, such as [2], or assume knowledge of the link lifetimes via an oracle, as in [4]. In terms of forming routes in wireless networks, it has long been argued that the "shortest path is not enough" [6], and therefore, the proposed methods are intended as a tool for serving the purpose of using alternate objectives when constructing routes. ${ }^{2}$

\footnotetext{
${ }^{1}$ Either direct exclusion of existing radio links or division of the existing links in "good" and "bad," where "good" links are favored, and "bad" links are used only when necessary.

${ }^{2}$ Alternate is to be understood in the sense of either 1 ) using only the proposed metrics or 2) combining them with traditional metrics (e.g., hop count).
}

Our contributions include the development of a "black box" method, which is intended to provide a tool that can be utilized to increase the robustness of protocols and schemes against rapid topology changes and short-lived links. The advantages of this method over existing work, which is based on signalto-noise ratio (SNR) or received power measurements, include the following: 1) the potential to acquire estimates over the whole duration of the link for both improving and deteriorating link quality; 2) accurately capturing the effect of the minimum distance achieved between the nodes on the link's lifetime; 3) accurately capturing the rate of change of the separating distance between the nodes, which is not always constant, even for constant relative speed between the nodes; and 4) offering the possibility to lift the assumption of knowledge of the transmission power or the path loss exponent. To the best of our knowledge, our study is the first to explicitly evaluate the adverse effect of shadow fading on the estimations. In addition, we present a framework that analytically quantifies the comparison capabilities of the estimates based on the accuracy level (i.e., on the probability distribution function of the error).

The rest of this paper is organized as follows: Section II gives an overview of related work, and the framework for estimating LRT is presented in Section III. More specifically, the problem is formulated in Section III-A, where we elaborate on the desired attributes of the solution. We continue to study the underlying structure of vehicle movement in Section III-B and derive the LRT estimation model in Section III-C. The scheme for analyzing the capability of correct identification of the link, which has the longest LRT among two given links, is presented in Section IV. We evaluate the accuracy and identification capabilities of the proposed method, comparing it with affinitybased schemes in Section V. Section VI continues to propose how the LRT estimates can be used for VANET routing and evaluates the effectiveness of the method for route construction, isolating it from route maintenance, when optimizing for longer lifetime. To this end, we present and use a modified version of Dijkstra's algorithm to find max-min paths in a connected graph. This paper concludes with Section VII.

\section{RELATED WORK}

The problem of routing through paths of higher lifetime, which entails the estimation of the links' lifetimes and the routing decision process, has been the topic of several recent studies. As far as the link lifetime estimation methods are concerned, related studies can largely be divided into three categories: 1) methods that assume knowledge of the other nodes' positions and velocity vectors (such as [2], [7], [8], or [9]);2) analytical methods based on the extraction of lifetime distributions (such as [10] or [11]); and 3) methods that make use of some link quality metric (such as [12]-[16] or [17]).

In general, methods of the first category require use of additional equipment, whereas the transmission range of the nodes is considered deterministic and known a priori, assuming a free space propagation model and a fixed known value of transmission power. However, being within range, as this is 
specified based on this assumption, does not always guarantee radio connectivity, particularly in an urban environment. The methods proposed here include transmission power as a parameter, which is to be estimated by the methods themselves. Moreover, the information acquired via GPS might be inaccurate. Methods targeting at analytically extracting link lifetime distributions naturally operate under the assumption of specific mobility models, such as the random waypoint model, and are thus applicable only to these cases when the vehicles follow the mobility model, on which the analysis has been based on. Utilizing information related to the quality of the radio link to construct reliable routes offers an immediate way to exploit the direct coupling of physical layer operations to the network topology. There exist approaches, such as [12], [13], or [14], employing the exponentially smoothed value of the received power or SNR of ongoing packets. Such a criterion focuses more on the past observations without extrapolating in the future, and thus implicitly assumes a stable grouping of the nodes, and the existence of neighbors with similar mobility characteristics, which may not always be the case in such a dynamic environment. Although SNR is a strong indicator of the instantaneous link quality and may thus, under certain conditions, imply larger momentary throughput, utilizing the smoothed SNR as a metric for route construction does not necessarily lead to longer-living routes (see Section VI-B).

Different approaches, such as [15], [16], or [17], make use of the time derivative of the received power or SNR of the monitored packets. These methods may successfully capture the temporal trend of the link quality but cannot always be used to make an informed choice between two given links. In addition, the temporal dependence of link quality is not linear, as dictated by the path loss exponent and the underlying structure of vehicle movement. Finally, explicit use of the time derivative is very sensitive to noise, which will inherently affect channel measurements due to multipath and shadow fading. Given the challenging propagation environment in which VANETs are operating, using the instantaneous rate of the link quality to extrapolate into the future may lead to erroneous estimates. We elaborate more on the technical details and deficiencies of affinity-based methods in Section V-C.

\section{FRAMEWORK FOR LINK RESIDUAL-TIME ESTIMATION}

\section{A. Problem Formulation and Analysis}

Assuming a given radio link between nodes moving relatively to one another, the link quality on the large scale will successively improve (improving phase), reach a maximum value, and then start deteriorating (deteriorating phase) until communication is no longer possible. We have defined LRT as the remaining time that a given link will continue to be useful for satisfactory data transmission. This translates into the residual time for which the link quality on the large scale should continuously remain above a specified threshold. We take a cross-layer approach, where physical layer knowledge is utilized by having the nodes monitor the link quality on incoming and overheard data packets, pilot symbols, and/or acknowledgement packets so that a time series is formed at every node for each of its neighbors. This way, no overhead in terms of traffic is introduced in the network. The problem is formulated as follows: Given the time series of a link quality metric $\left(t_{j}, S\left(t_{j}\right)\right)$ on a specific link, estimate the remaining time before the link quality $S(t)$ drops below the specified threshold. We address this problem as a parameter estimation problem: The parameters we want to derive out of the SNR samples are related to large-scale path loss, treating random variations on the received power level as noise. The estimated parameters can then be used to predict the time point when the link quality on the large scale will drop below the specified threshold and, thus, produce estimates of the LRT. In the following, we elaborate on the desired attributes of an LRT-related metric, describe specific challenges arising, and summarize the way we have addressed the relevant requirements.

First, since we want to derive the distance-dependent part of the path loss, multipath and shadow fading introduce considerable noise in the collected time series, hindering the accurate extraction of the desired parameters. We aim to produce methods robust to these sources of noise. The use of the time derivative, as proposed in existing work, may be highly problematic; as will be pointed out in Section V-C, the performance of existing affinity-based schemes significantly drops when considering multipath fading. To the best of our knowledge, this paper is the first approach to explicitly evaluate the performance of the developed estimation methods under various scenarios of channel fading. Furthermore, the fact that we are only able to sample the channel condition when the link is active ${ }^{3}$ results in limited knowledge of the underlying signal. For these two reasons, our methods utilize as much of the collected data as possible via nonlinear regression, contrary to existing methods that rely on instantaneous data, and are thus more sensitive to deep fades in the channel link quality.

Second, since we aim to use the link lifetime estimations for optimizing the route lifetime, the proposed metric needs to go beyond describing the tendency of the future quality of given links and be capable of capturing the remaining link lifetime as accurately as possible. For this reason, we employ a model that exploits the underlying characteristics of radio propagation and vehicle movement. We introduce a model describing the temporal dependence of link quality, which allows for the inclusion of the following factors: 1) the fact that link quality does not vary linearly with the separating distance between the nodes, but the respective rate of change is dictated by the path loss exponent, and 2) the fact that when the nodes no longer move along the line defined by their positions, the rate of change of their separating distance does not equal the relative speed between them.

Most importantly, the nature of route construction implies that the metric should ideally support the possibility of making an informed choice between any two given links. The main advantage of the model presented here is that it can be used to produce estimates of the LRT even during the improving phase of a radio link. Existing work using the time derivative of the link quality metric (affinity-based schemes, such as [15]

\footnotetext{
${ }^{3}$ Either through transmitted packets or pilot symbols and receipt acknowledgements.
} 
or [16]) implicitly assumes that any link in an improving phase is more reliable than a link in a deteriorating phase. This, however, is not true as the link reliability also depends on the relative speed between the nodes and on the peak value of the received power or SNR. Elaborating on the latter, the remaining link duration during the improving phase is conditional on the time point when the link quality starts deteriorating (which is the time point on which the peak value of the received power or SNR will be accomplished). In [17], a method, based on utilization of the signal strength changing rate, is presented, predicting the remaining time even during the improving phase of the link, but the model used assumes that the minimum distance that will be achieved among the two nodes is zero, whereas the transmission range and the path loss exponent are considered known. The improved accuracy of the proposed estimation method stems from the incorporation of the minimum distance achieved during the nodes' movement as a parameter in the link quality model used. This enables us to extrapolate on the future throughout of both phases.

\section{B. Underlying Structure of Vehicle Movement}

The proposed method's increased accuracy and the model's ability to quantify the links' residual time even during the improving phase lie in the incorporation of the minimum distance achieved during the nodes' movement as a parameter to be estimated by the method. The main difference with respect to our previous work in [18]-[20] is that the model developed here is used to derive an estimate of the minimum distance between the nodes, even before this has been achieved, i.e., before the maximum value of the received power has been reached. This is of crucial importance as knowledge of the time point when the link quality reaches a maximum value enables us to extrapolate the future value of received power on the large scale even throughout the improving phase of the link. We achieve this by using the Pythagorean theorem to express the physical distance between the nodes as the sum of the squares of the minimum distance achieved and the distance of the mobile from the origin of a specific axis. The axis is chosen so that the distance of the mobile from the origin linearly varies with the mobile's speed, thus integrating the temporal dependence on the link quality model.

Due to the nature of the problem, it is the large-scale phenomenon (path loss) that we are trying to capture and predict, namely, the change in channel quality (either attenuation or amplification) caused by the change in the relative distance between sender and receiver. However, the distance between the transmitter and the receiver is not assumed to be known to the individual nodes. In contrast, what we have available is a time series of samples of SNR and the time points when these were monitored, i.e., $\left(t_{i}, S_{i}\right)$. Hence, we need a model that employs time as the independent variable. To this end, we are proceeding to acquire a model for the dependence of the separating distance with time. Let us consider two mobile nodes $a$ and $b$, as shown in Fig. 1(a). As explained, we are interested in acquiring an expression of the temporal dependence of the distance between them as they are moving on the $x y$ plane. Let $\mathbf{v}_{a}=\left(v_{a, x}, v_{a, y}\right)$ and $\mathbf{v}_{b}=\left(v_{b, x}, v_{b, y}\right)$ denote the velocity vectors of mobile

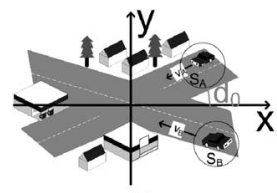

(a) (b)

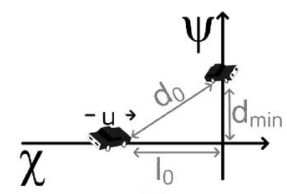

Fig. 1. Relative motion of two mobile nodes $a$ and $b$.

nodes $a$ and $b$, respectively. ${ }^{4}$ In addition, let $\mathbf{S}_{a, 0}=\left(x_{a, 0}, y_{a, 0}\right)$ and $\mathbf{S}_{b, 0}=\left(x_{b, 0}, y_{b, 0}\right)$ denote the position vectors of the nodes at time $t_{0}$, and let $d_{0}=\left|\mathbf{S}_{a, 0}-\mathbf{S}_{b, 0}\right|$ denote the initial distance between them. It follows that the temporal dependence of the separating distance between the nodes is given by

$$
d(t)=\sqrt{\left(\Delta x_{0}+\Delta v_{x}\left(t-t_{0}\right)\right)^{2}+\left(\Delta y_{0}+\Delta v_{y}\left(t-t_{0}\right)\right)^{2}}
$$

where $\Delta k_{0}=k_{a, 0}-k_{b, 0}$, and $\Delta v_{k}=v_{a, k}-v_{b, k}$, with $k=$ $\{x, y\}$. Based on (1), the minimum distance that will be achieved between the nodes during their movement depends on their initial positions and their velocity vectors and is given for $t_{m}=-\left(\Delta x_{0} \Delta v_{x}+\Delta y_{0} \Delta v_{y} /\left(\Delta v_{x}\right)^{2}+\left(\Delta v_{y}\right)^{2}\right)+t_{0}$ as $^{5}$

$$
d_{\min }=d\left(t_{m}\right)=\sqrt{\frac{\left(\Delta y_{0} \Delta v_{x}-\Delta x_{0} \Delta v_{y}\right)^{2}}{\left(\Delta v_{x}\right)^{2}+\left(\Delta v_{y}\right)^{2}}} .
$$

The minimum distance that will be achieved between the nodes, which is usually considered negligible in similar studies, is a factor that affects the link lifetime significantly. As an example, we note here that for any value of relative speed, the link duration increases by $80.28 \%$ of its value when the minimum distance achieved changes from 100 to $10 \mathrm{~m}$ (for a range of approximately $120 \mathrm{~m}$ ).

Based on (1), we proceed to derive the relationship between the separating distance between the nodes over time and $d_{\text {min }}$. Without loss of generality, we consider node $a$ to be stationary; node $b$ 's motion will then be specified relative to node $a$. We are performing two consecutive transformations ${ }^{6}$ in the Cartesian plane so that node $b$ moves on the horizontal axis and node $a$ resides on the vertical axis [see Fig. 1(b)]. This means that the minimum distance will be achieved when node $b$ passes through the origin. Node $b$ 's motion will now be described by the velocity vector $\mathbf{u}=\left(u_{b, x}, u_{b, y}\right)=\left(\sqrt{\left(v_{b, x}\right)^{2}+\left(v_{b, y}\right)^{2}}, 0\right)$. Based on the coordinate system in Fig. 1(b), we obtain the following relationship for the temporal dependence of the distance:

$$
d(t)=\sqrt{d_{\min }^{2}+\left(u_{b, x}\left(t-t_{0}\right)-l_{0}\right)^{2}}
$$

\footnotetext{
${ }^{4}$ For the following, we are assuming that the velocity vectors remain constant and that at time $t_{0}$ the two nodes are approaching each other.

${ }^{5}$ The first derivative of the separating distance with respect to time is equal to $\theta d(t) / \theta t=\left(\Delta x_{0}+\Delta v_{x}\left(t-t_{0}\right)\right) \Delta u_{x}+\left(\Delta y_{0}+\right.$ $\left.\Delta v_{y}\left(t-t_{0}\right)\right) \Delta v_{y} / d(t)$. The distance is minimized at time point $t_{m}$, as $\left.(\theta d(t) / \theta t)\right|_{t_{m}}=0$, and $\left.\left(\theta^{2} d(t) / \theta t^{2}\right)\right|_{t_{m}}=\left(\left(\Delta v_{x}\right)^{2}+\left(\Delta v_{y}\right)^{2} / d_{\min }\right)>$ 0 . It should be noted that $t_{m}$ is strictly positive based on the fact that we have assumed the nodes to be approaching each other at $t_{0}$; thus, the temporal derivative of the distance at $t=t_{0}$ should be negative: $\left.(\theta d(t) / \theta t)\right|_{t_{0}}=\left(\Delta x_{0} \Delta v_{x}+\Delta y_{0} \Delta v_{y} / d_{0}\right)<0$.

${ }^{6} \mathrm{~A}$ rotation so that $b$ moves parallel to the $x x^{\prime}$ axis and a translation so that the movement coincides with the $x x^{\prime}$ axis. This results in node $a$ residing on the vertical axis.
} 
where $l_{0}$ is the distance of node $b$ from the beginning of the axis system shown in Fig. 1(b) at time $t_{0}$ (the time when the link between the nodes was established). We note that (3) is indicative of the fact that the separating distance will only vary linearly with time when $d_{\min } \rightarrow 0$.

\section{Link Residual-Time Estimation Model}

A widely used model (see, for example, [21]) for the received power on a wireless link is the following:

$$
P_{R}(d)=P_{0}-10 \gamma \log (d)+\phi_{R}+\phi_{S}(\mathrm{dBm})
$$

where $P_{0}$ denotes the received power at a reference distance $d_{r}=1 \mathrm{~m}$, depending on the transmitting power and the gains of the transmitting and receiving antenna, and $\gamma$ is the path loss exponent factor, depending on the environment. The variations caused by multipath fading are denoted by $\phi_{R}$, whereas $\phi_{S}$ represents the changes on the level of the received power due to shadow fading. Assuming additive white Gaussian noise with a mean power of $N_{0}(\mathrm{dBm})$, the SNR can be modeled with respect to the nodes' separating distance $d$ as

$$
\operatorname{SNR}(d)=P_{0}-10 \gamma \log (d)+\epsilon-N_{0}=\mathrm{SNR}_{0}-10 \gamma \log (d)+\epsilon
$$

where $\mathrm{SNR}_{0}$ denotes the $\mathrm{SNR}$ on the large scale at a reference distance $d_{r}=1 \mathrm{~m}$. Shadow- and multipath-fading-induced variations are denoted by $\epsilon$ to indicate that our method does not aim to derive estimates of these parameters. As LRT is calculated on a higher timescale, random channel variations are considered to introduce noise in the collected time series, hindering the accurate extraction of parameters related to LRT estimation. Combining (3) and (5), we derive the following model for the temporal dependence of SNR on the large scale (i.e., without taking random fluctuations into account):

$$
\operatorname{SNR}(t)=\operatorname{SNR}_{0}-5 \gamma \log _{10}\left(d_{\min }^{2}+\left(u_{b, x}\left(t-t_{0}\right)-l_{0}\right)^{2}\right) .
$$

Solving for $t$ when $\operatorname{SNR}(t)=\mathrm{SNR}_{\mathrm{th}}$, where $\mathrm{SNR}_{\mathrm{th}}$ is the SNR value below which the link cannot be used for communication, (6) gives the LRT as

$$
\operatorname{LRT}(t)=\frac{\sqrt{10^{\left(S N R_{0}-S N R_{\mathrm{th}}\right) / 5 \gamma}-d_{\min }^{2}}+l_{0}}{u_{b, x}}-\left(t-t_{0}\right) .
$$

The method comprises of using the time series to regress on (6), acquiring the parameters that dictate the change in SNR on the large scale and plugging them in (7) to acquire the estimates of the LRT. The nonlinear least-square estimation method used to acquire the estimates of the parameters is a subspace trust region method based on an interior reflective Newton method. The nonlinear least-square regression method utilizes (6) and the collected time series, where time and SNR are the independent and dependent variables, respectively. The parameters that are to be estimated are the received SNR at the reference distance $\mathrm{SNR}_{0}$, the minimum distance achieved $d_{\text {min }}$, the relative speed between the nodes $u_{b, x}$, the initial position of node $b$ on the $\chi \chi^{\prime}$ axis $l_{0}$, and the path loss exponent $\gamma$.

\section{IDENTIFICATION CAPABILITY FRAMEWORK}

When using the LRT estimation method for the purpose of routing, the merit of evaluating the proposed model does not lie so much on the absolute accuracy achieved but rather on the estimates' capability of comparing different links and correctly identifying the one that will last longer, i.e., the one with the largest LRT. In this section, we are presenting a framework that may be used to analytically quantify the identification capabilities of an LRT estimation method based on the probability distributions of the estimation errors, i.e., on the analytical expression of the method's accuracy in capturing LRT.

For the following, let $T_{m}$ and $T_{n}$ denote the actual LRTs of two links $m$ and $n$, and let $\tilde{T}_{m}$ and $\tilde{T}_{n}$ denote the corresponding estimates. The uncertainty in the LRT estimates can be represented by a random variable that follows the distribution of the error. We therefore define a family of random variables, which is denoted by $E_{k}: \Omega_{E_{k}} \rightarrow \mathbb{R}$. $^{7}$ It follows that the estimates themselves are random variables, i.e., as the sum of two random variables

$$
\tilde{T}_{k}=T_{k}+E_{k}
$$

We are then interested in the probability that the LRT estimates will maintain the relative order of the actual LRTs. We define a function $I: \Omega_{T} \times \Omega_{T} \rightarrow[0,1]$, i.e.,

$$
I(m, n)= \begin{cases}\operatorname{Pr}\left(\tilde{T}_{m}>\tilde{T}_{n}\right), & \text { if } T_{m}>T_{n} \\ \operatorname{Pr}\left(\tilde{T}_{m}<\tilde{T}_{n}\right), & \text { if } T_{m}<T_{n} \\ 1, & \text { if } T_{m}=T_{n}\end{cases}
$$

which gives the probability of taking the correct decision, when using the LRT estimates with the aim of choosing the link of the longest actual LRT, between links $m$ and $n$. Based on the distribution of the errors $f_{E_{k}}$, we can calculate the value of $I$. Without loss of generality, let us examine the case $T_{m}>T_{n}$, i.e.,

$$
I(m, n)=\operatorname{Pr}\left(\tilde{T}_{m}>\tilde{T}_{n}\right)=\int_{T_{n}-T_{m}}^{\infty} f_{m-n}(u) d(u) .
$$

Given the distributions $f_{E_{k}}$, the distribution of the difference of the errors $E_{m}-E_{n}$, which is denoted by $f_{m-n}$, is equal to the cross correlation of their distributions, i.e.,

$$
f_{m-n}(u)=\int_{-\infty}^{\infty} f_{n}(\tau) f_{m}(\tau+u) d \tau=f_{n} \star f_{m} .
$$

It follows that the lifetime of the link chosen based on the estimates of LRT is also a random variable, which is denoted by $L$. The conditional expected value of the lifetime of the chosen link $L$ on the actual LRT values of the candidate links $T_{n}$ and $T_{m}$ is given by

$$
\begin{aligned}
E\left[L \mid T_{n}, T_{m}\right]=I(m, n) & \cdot \max \left(T_{m}, T_{n}\right) \\
+ & {[1-I(m, n)] \cdot \min \left(T_{m}, T_{n}\right) . }
\end{aligned}
$$

\footnotetext{
${ }^{7}$ The index $k$ indicates the error distribution for $T=T_{k}$
} 
Based on this, we can now calculate the expected value of the lifetimes of the links chosen based on the LRT estimation method as

$$
\begin{aligned}
E\left[L_{\mathrm{LRT}}\right]= & \int_{\Omega_{T}} \int_{\Omega_{T}}\left[I(m, n) \cdot \max \left(T_{m}, T_{n}\right)\right. \\
& \left.\quad+(1-I(m, n)) \cdot \min \left(T_{m}, T_{n}\right)\right] \\
& \cdot f_{T}\left(T_{m}\right) f_{T}\left(T_{n}\right) d T_{m} d T_{n} .
\end{aligned}
$$

To numerically evaluate the optimality of the method, we also calculate the expected value of the lifetimes had the links been chosen based on an oracle, which would always opt for the longest living link, i.e.,

$$
E\left[L_{O}\right]=\int_{\Omega_{T}} \int_{\Omega_{T}} \max \left(T_{m}, T_{n}\right) f_{T}\left(T_{m}\right) f_{T}\left(T_{n}\right) d T_{m} d T_{n}
$$

as well as the expected value of all residual lifetimes to serve as a benchmark that represents the average case, i.e.,

$$
E[L]=\int_{\Omega_{T}} t f_{T}(t) d t .
$$

\section{VALidation OF Link Residual Time Estimation}

\section{A. Technical Details}

For the following, we are considering a link between two nodes, one of which is moving relative to the other, as shown in Fig. 1(b). As we have shown, this scenario can adequately describe the change in the separating distance of two nodes moving on the plane, each maintaining a constant speed. The received power at the reference distance is taken to be equal to $P_{0}=-17 \mathrm{dBm}$, whereas the noise level is considered to be at $N_{0}=-95 \mathrm{dBm}$, and the threshold for efficient communication is set at $\mathrm{SNR}_{\mathrm{th}}=5 \mathrm{~dB}$. These values are consistent with the assumption of $802.11 \mathrm{~g}$, resulting in a range of approximately $120 \mathrm{~m}$. We are assuming that any packet above $0 \mathrm{~dB}$ can be detected and therefore used by the method. We assume that physical layer information is passed on to the algorithm every $T=250 \mathrm{~ms}$ in the form of the mean SNR over all packets received on that link during this time interval. When there is no traffic on the link, or when the data packet interval is larger, the method can still make use of the "hello" packets, which are an integral part of ad hoc network protocols. However, when the instantaneous channel conditions cause the received power of all the transmitted packets to fall below the receiver's detection threshold, there is no data available, and the estimation method uses previously collected data. The latter is captured in the simulation results presented in the following. The main rationale for examining the performance of the method when averaging the SNR samples over $T$ is that in practice the method would be rather computationally demanding if it were applied every time a new packet is received. The packets are sent at random time points with a rate of $\lambda=0.1$. The path loss exponent $\gamma$ is taken to be equal to 3.5 .

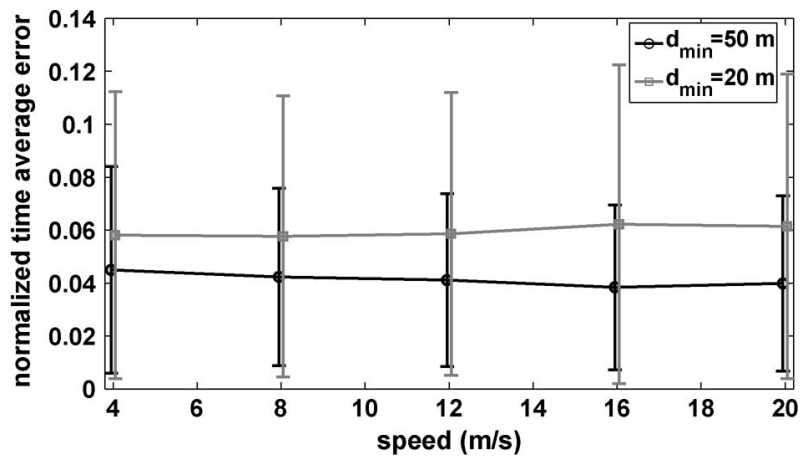

Fig. 2. Normalized error versus speed for different values of $d_{\min }$.

For the case of multipath fading, the envelope of the received power is assumed to follow a Rayleigh distribution for nonlineof-sight conditions or a Rician distribution in the case when there exists a line of sight between transmitter and receiver. It must be noted that the Rician distribution can be controlled by the Rician $k$ factor, which is a parameter that controls the ratio of the average received power via the line-of-sight path versus the average power received through other paths. For the simulations, multipath fading is modeled via use of a filter that produces the Jakes Doppler spectrum [22], which is consistent with the predictions of the widely used Clarke's model for the mobile radio channel. For implementation details, see [23].

When modeling shadow fading, it is important to take the spatial cross correlation into account. The correlation distance (which is denoted by $L_{C}$ ) under a mobile node(s) scenario is indicative of the (temporal) rate of change of the shadowing level and depends on the sizes of the obstacles, i.e., other vehicles, buildings, trees, etc. For the following simulations, we follow the approach taken in [24], exploiting the fact that interpolation is a standard way of introducing correlation. We generate uncorrelated values drawn from a zero-mean Gaussian distribution with the desired standard deviation $\sigma$ assuming that the sample spacing is equal to the correlation distance. We then perform interpolation between these samples, introducing correlation to the uncorrelated generated values, to obtain the value of shadow fading at required locations. The interpolation can take place down to a specific sampling rate to generate the required time series or only on the separating distance corresponding to the time of data transmission, thus enabling efficient integration to a large-scale simulation, as in Section VI-B.

\section{B. Estimation Accuracy}

In this section, we briefly present the results related to the accuracy of the estimation methods to demonstrate how different characteristics of the vehicles' movement might affect the error in the estimates of LRT. Fig. 2 shows the normalized time average error in LRT estimation as a function of the relative speed between the nodes for different values of $d_{\min }{ }^{8}$ To assess the accuracy of the estimates, we are using the mean ${ }^{9}$ time average ${ }^{10}$ error normalized by the link duration.

\footnotetext{
${ }^{8}$ Each point shown has been averaged over 20 different Monte Carlo iterations of a Rician fading channel.

${ }^{9}$ Over different Monte Carlo iterations.

${ }^{10}$ Over the successive estimates.
} 


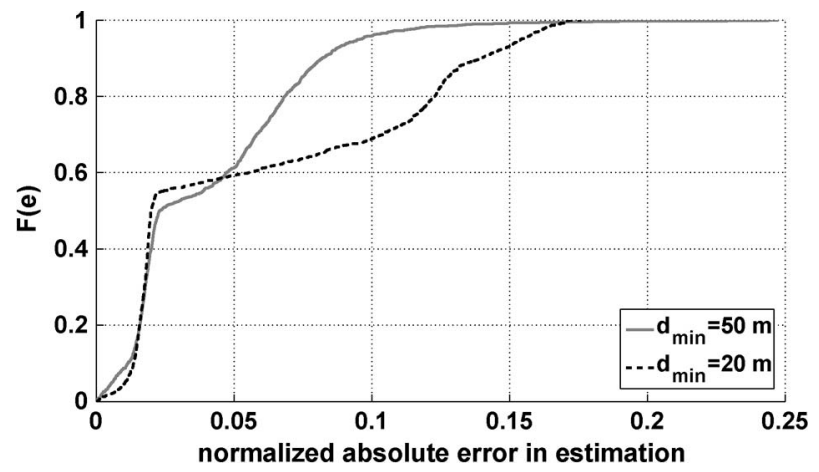

Fig. 3. Empirical cdf for the normalized errors when $u=12 \mathrm{~m} / \mathrm{s}$.

In addition, we plot bars indicating the standard deviation of the normalized error. We note that although the mean value of the normalized error is around $6 \%$ for $d_{\min }=20 \mathrm{~m}$ and $4 \%$ for $d_{\min }=50 \mathrm{~m}$, the corresponding median values are smaller than $2 \%$ and $3 \%$, respectively. This indicates a higher variability for $d_{\min }=20 \mathrm{~m}$. The variability in the accuracy of the estimates can be seen in Fig. 3, where we plot the empirical cumulative distribution function (cdf) of the normalized errors acquired during the whole duration of the link for 20 Monte Carlo iterations, when the relative speed is $12 \mathrm{~m} / \mathrm{s}$. This variability in the accuracy is due to different Monte Carlo iterations while also indicative of the fact that the accuracy will initially fluctuate until a sufficient number of samples has been acquired. In general, the relative speed does not seem to affect the relative accuracy significantly. The absolute error, however, drops for larger values of the relative speed as the temporal dependence of the SNR becomes more evident faster and thus is easily detectable with fewer SNR samples. It should also be noted that the number of estimations that have taken place may considerably differ when examining links among mobiles whose relative motion is described by different parameters $\left(d_{\min }, u_{b, x}\right)$.

\section{Identification Capabilities}

We are comparing the identification capabilities of the LRT estimates with an affinity-based scheme (such as in [15] or [16]), which makes use of the time derivative of the SNR (or signal strength). The affinity between two nodes, which is denoted by $n$ and $m$, is given by

$$
a_{n, m}=\left\{\begin{array}{l}
\text { high if } \Delta S_{n, m}>0 \\
\frac{S N R_{\mathrm{th}}-S_{\mathrm{curr}}}{\Delta S_{n, m} / \Delta t}, \text { otherwise }
\end{array}\right.
$$

where $S_{\text {curr }}$ is the currently reported SNR at the link in question, and $\Delta S_{n, m} / \Delta t$ is the rate of change of the SNR on the link, averaged over the last few samples. As has already been mentioned, affinity-based schemes do not quantify the link lifetime when the nodes are approaching each other and cannot therefore be used to make an informed decision between two links that are both in an improving phase (positive changing rate of SNR). However, the relative velocity vectors and the minimum distance achieved vary between different pairs of nodes; thus, it is not guaranteed that a link going through an improving phase will last longer than one whose link quality is decreasing. Although the rate of change of the link quality metric may successfully capture the tendency of a link's condition, we have found that when used to compare two links, it can lead to unfair conclusions. The rate of change of the SNR is not constant throughout the duration of the nodes' movement (that is, SNR will not linearly change with time). When measured in watts, we have SNR $\propto\left(1 / d(t)^{\gamma}\right)$ [25], where $d(t)$ is the separating distance between the nodes, which varies with time. ${ }^{11}$ It follows that the rate of change of the SNR will vary with time according to $\Delta(S / \Delta t) \propto$ $-\left(\gamma d^{\prime}(t) / d^{\gamma+1}(t)\right)$. Similarly, measured in decibels, we will have $(\Delta S / \Delta t) \propto-\left(\gamma d^{\prime}(t) / d(t)\right)$. When using the current rate of change to extrapolate into the future, links of nodes that are closer physically will be characterized by a smaller affinity than they should. In addition, the fact that affinity is not quantified for positive values of the rate of change may be problematic. Depending on the relative speed between nodes or the minimum distance that will be achieved between them, a link $l_{a}$ such that $\Delta S_{l_{a}}>0$ may have a much smaller residual lifetime than a link $l_{b}$ with $\Delta S_{l_{b}}<0$. Finally, the explicit use of the time derivative severely decreases the reliability of the estimates under the variations caused by channel fading. We will now demonstrate these deficiencies through comparing the identification capabilities of the proposed method and affinitybased decisions. We are also presenting the lifetimes achieved under a scheme agnostic to LRT (13) and the lifetimes achieved under an oracle (14) to serve as benchmarks.

To calculate $\Delta S_{n, m} / \Delta t$, we follow [15], where $N$ previous samples are used to calculate the mean rate of SNR with respect to the current sample. Since the previous studies do not identify a specific value for $N$ (the number of previous samples used for affinity calculation), we present the results for $N$ ranging between 1 and 20 . The value of $N$ is restricted by the duration of the links under examination. ${ }^{12}$

We produce LRT estimates and calculate the affinity every $250 \mathrm{~ms}$ for each link ${ }^{13}$ during the whole link duration (when $\mathrm{SNR} \geq \mathrm{SNR}_{\mathrm{th}}$ ). All the estimates produced during the duration of the link are used. Links are chosen based on the calculated affinity as follows: If both links are in an improving phase (high affinity) we choose randomly, then links of high affinity are always preferred over links with deteriorating link quality, whereas when the affinity is not high for any of the two links, we opt for the link of higher affinity value. The LRT estimation method uses the model described in (6) and the time series of the received power of collected packets real-time to produce estimates of five parameters: 1) the SNR at a reference distance $\left.S N R_{0} ; 2\right)$ the path loss exponent $\gamma ; 3$ ) the minimum distance

\footnotetext{
${ }^{11}$ The dependence of the separating distance with time is not always linear but depends on $d_{\min }$.

${ }^{12}$ Since the calculation of the derivative should be limited to either improving or deteriorating phase to give meaningful results, $N$ cannot cover more than the duration of a single phase and, ideally, should not extend to more than one phase. The value of $N=20$ is equivalent to $5 \mathrm{~s}$, since we take samples every $250 \mathrm{~ms}$. We note that the phase durations for the scenarios examined reach down to this value.

${ }^{13}$ We are simulating mobile radio links for different scenarios of the nodes' movement. The relative speed between the nodes varies between 2 and $20 \mathrm{~m} / \mathrm{s}$, whereas the minimum distance achieved ranges from 10 to $100 \mathrm{~m}$.
} 
TABLE I

EXPECTED VALUES OF LIFETIMES

\begin{tabular}{|r|ccc|}
\hline Method & Ideal Channel & Rician fading & Rayleigh fading \\
\hline Optimal & 41.92 & 41.01 & 37.82 \\
\hline$L R T$ & 41.91 & 40.56 & 37.01 \\
\hline Affinity $N=1$ & 36.10 & 29.02 & 25.65 \\
$N=5$ & 35.93 & 31.28 & 26.91 \\
$N=10$ & 35.73 & 32.25 & 27.72 \\
$N=20$ & 35.35 & 32.92 & 28.36 \\
\hline Random & 27.67 & 27.04 & 24.90 \\
\hline
\end{tabular}

achieved $d_{\min }$; and 4) the relative speeds of the nodes $u_{b, x}$ and $l_{0}$. These estimates, which are produced every $T$ seconds, are plugged in (7) to produce the LRT estimates used as a factor to choose the longest living link. In total, the pairs of links used are in the order of $55 \cdot 10^{6}$.

Table I shows the expected values of the lifetimes achieved under different decision mechanisms for different channel conditions. First, we evaluate the efficiency of the methods in the (ideal) situation when the channel is affected by neither multipath nor shadow fading to illustrate the suitability of the proposed model as a route construction criterion and to enable comparison with other methods when the results are not affected by noise. The packets are assumed to be sent instantly every $T=250 \mathrm{~ms}$. We can see that the identification capabilities of the LRT estimates acquired under the proposed model (6) are practically identical with that of an oracle that always opts for the longest living link. This reflects the suitability of the metric for utilization in route construction functions. The affinity-based scheme, however, fails to consistently identify the longest living link among two, resulting in a much smaller mean lifetime value than the LRT-based decision. For ideal channel conditions, the best value for $N$ seems to be 1 , meaning that it is preferable to calculate the changing rate over the last pair of observations only, avoiding the use of stale information.

We can also see that the LRT-based decision is robust to noise, achieving to choose links of average lifetime equal to 98.9\% (under Rician fading) and $97.9 \%$ (under Rayleigh fading) with respect to $E\left[L_{O}\right]$. The affinity scheme is, as expected, very sensitive to the noise induced in the measurements by multipath fading due to the explicit use of the time derivative. We can see that the lifetimes achieved under the affinity-based scheme are much closer to the corresponding value given by an agnostic scheme (random decision) than they are at the optimal. In addition, the merit of using more samples to calculate the changing rate is now evident. As opposed to the results under ideal channel conditions, the affinity-based scheme seems to perform better for bigger values of the parameter $N$, which controls the number of samples used for the time derivative calculation. However, as has been mentioned, the value of $N$ is restricted by the link duration. To evaluate the merit of utilizing a link lifetime criterion, we note that the proposed LRT estimation method results in the choice of links that last $50 \%$ or $48.7 \%$ longer when compared with an agnostic scheme for Rician or Rayleigh fading, respectively. The corresponding values for the affinity-based scheme are $21.8 \%$ and $13.9 \%$ (calculated for the best-case scenario $N=20$ ).
Shadow fading introduces significant challenges on the estimations, as particularly for smaller values of the relative speed between the nodes, and during the initial phase of the link, it may dominate the temporal dependence of the channel's quality. This hinders the estimation algorithm from accurately extracting the parameters of distance-dependent path loss.

Fig. 4(a) plots $E\left[L_{\mathrm{LRT}}\right]$ (12), as a function of $T_{n}$ and $T_{m}$, normalized by $E\left[L_{O}\right] \cdot{ }^{14}$ In addition, we plot the joint probability density function (pdf) $f_{T T}\left(T_{m}, T_{n}\right)$ for the corresponding values of $T_{m}$ and $T_{n}$ in Fig. 4(b) to illustrate how common the appearance of each pair is. We can see that the estimation methods achieve satisfactory results; for most of the pairs, the ratio approaches 1 . There is a drop in performance when comparing links of residual lifetime close to $110 \mathrm{~s}$ with links of smaller LRT. This phenomenon arises due to two main reasons: First, the difference of the actual LRTs of the two links is large, and therefore, even a small probability of misjudegment has an adverse effect. Second, the existence of links whose total lifetime was close to $110 \mathrm{~s}$ means that the corresponding estimates of these specific links in this area were less accurate as they were based on very few samples. Finally, the joint pdf shows us that most pairs will be concentrated around values smaller than $20 \mathrm{~s}$, which means that the performance of the comparison estimates for these pairs will affect the global performance to a greater extent. To assess the effect of shadowing to the identification capabilities of both the proposed estimation method and the affinity-based scheme, we define the following metrics:

$$
\begin{aligned}
& m_{1}=\frac{E\left[L_{\mathrm{LRT} / \mathrm{AFF}}\right]-E[L]}{E[L]} \\
& m_{2}=\frac{E\left[L_{\mathrm{LRT} / \mathrm{AFF}}\right]}{E\left[L_{O}\right]}
\end{aligned}
$$

where $m_{1}$ gives the performance gain of using LRT estimation (or the affinity-based scheme) compared with the average case as a percentage, and $m_{2}$, which is defined as the identification efficiency, is indicative of the closeness of the corresponding method to the optimal solution. Fig. 5 plots these metrics with respect to shadowing standard deviation. ${ }^{15}$ The tendency of the gain to decrease as shadow fading becomes more dominant was expected, as a higher shadowing standard deviation means that the time series used for estimation will be noisier; thus, we expect the results to be less accurate. In all the cases, the proposed method achieves to reach more than $93 \%$ of the optimal value (as shown by $m_{2}$ ), whereas the gain with respect to the average case (metric $m_{1}$ ) is larger than $42 \%$ for all values of shadowing standard deviation. The metrics for the affinity-based scheme (for the best-case scenario for $N=20$ ) stay above $79 \%$ and $5 \%$, respectively.

\footnotetext{
${ }^{14}$ The results have been obtained based on 1000 Monte Carlo iterations of links between nodes whose relative speed varies between 2 and $20 \mathrm{~m} / \mathrm{s}$ and which will achieve a minimum distance that ranges from 10 to $100 \mathrm{~m}$. The channel is affected by Rician $(k=8)$ and shadow $(\sigma=2 \mathrm{~dB})$ fading.

${ }^{15}$ The pdfs have been calculated empirically based on simulating 400 different channel realizations of a Rician fading channel with $k=8$ and for values of shadow fading ranging from 1 to $4 \mathrm{~dB}$. The relative speed between the nodes is uniformly distributed between 2 and $20 \mathrm{~m} / \mathrm{s}$, whereas the minimum distance achieved ranges from 10 to $100 \mathrm{~m}$
} 


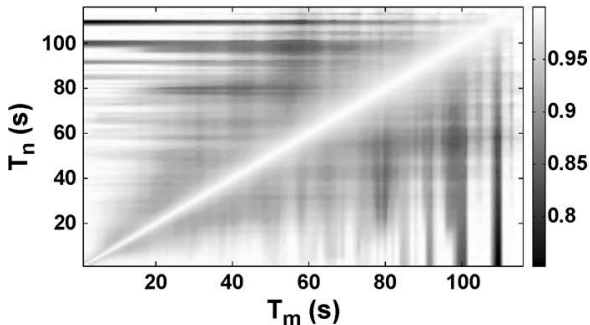

(a)

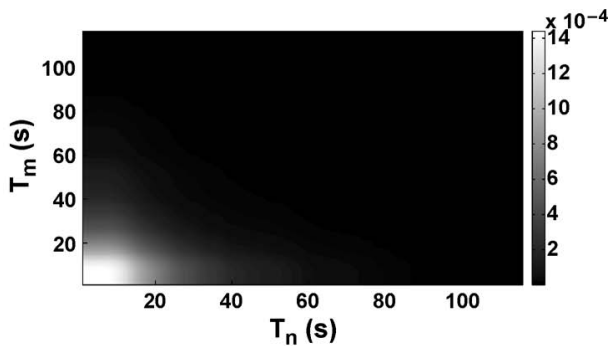

(b)

Fig. 4. Conditional expected value of the chosen link's residual time normalized by $\max \left(T_{m}, T_{n}\right)$ and the joint pdf of $T_{m}$ and $T_{n}$.

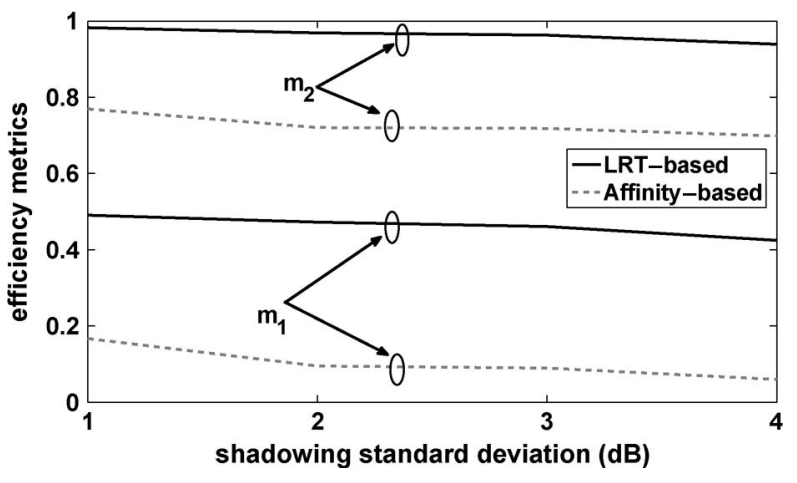

Fig. 5. Identification efficiency and performance gain metrics versus the shadowing standard deviation.

\section{Utilization of Link Residual Time ESTIMATES IN ROUTE CONSTRUCTION}

This section is intended to serve as an application scenario for the proposed method. We are examining the integration of the proposed LRT estimation method in route construction functions. We evaluate the efficiency of utilizing the estimates under the presence of shadow fading and assume that the path loss exponent is known based on previous measurements in the area that the network is operating. It should be noted that in this context, we are interested in evaluating the merit of the use of LRT estimations in the initial route construction procedure, isolating it from route maintenance. This obviates a frequent need for route reconstructions, which are costly in terms of bandwidth, achieving on the whole a better management of network resources. Although the metric proposed may be used in conjunction with other route construction metrics (e.g., expected throughput or minimum hop), examining the case when LRT estimates are used as a sole metric for acquiring the routing path allows us to demonstrate the true gain achieved. The inaccuracy of the estimates, induced mainly by shadow fading variations, implies that the estimated RRT will be less accurate for longer routes. In that sense, not constraining the number of hops allows us to demonstrate that despite the inaccuracies, the estimates are robust enough and can indeed be used as a route construction metric with the target of optimizing route lifetime.

Our focus is not on evaluating specific routing protocols but rather on comparing the lifetimes achieved under use of different metrics. The minimum hop count represents most existing VANET protocols, such as Ad hoc On Demand Distance Vector (AODV) protocol [26] or destination-sequenced distance-vector routing [27], whereas the use of the smoothed SNR is a commonly used metric for lifetime-based routing (e.g., [14]). The lifetimes of the routes presented here are the lifetimes that would be achieved under any VANET routing protocol using the metrics under discussion, as the result of the optimization would not change even if the specifics of the dissemination (e.g., integration in a reactive versus a proactive protocol) of routing information change. To this end, we have devised a modification to the classical Dijkstra's algorithm [28]. The resulting algorithm identifies the max-min path in a connected graph. The possibility to modify Dijkstra's algorithm to discover the longest living route implies that choosing the route that maximizes the minimum LRT among the links is not more complex than using a distance metric. It follows that any routing protocol using a distance metric can directly be modified to include LRT in its objective function. The results of [4] give strong evidence that, when evaluating with respect to route lifetime maximization, assuming the availability of link characteristics in the source for simulation gives the same insight as implementing a fully fledged routing protocol with the same objective function. Given a connected graph $G(t)=$ $(V, E(t))$, where $V$ denotes the set of vertices (mobile nodes) and $E$ denotes the set of edges (wireless links existing at time $t$ ), and an LRT function $l: E \rightarrow \mathbb{R}$ mapping edges (links) to their residual times, the modified Dijkstra's algorithm that is employed finds a max-RRT path from a source node $s \in V$ to a destination node $d \in V$, as shown in Fig. 6. A similar problem is studied in [4], where the algorithm proposed has a complexity of $O\left(N^{4}\right)$, where $N$ is the number of nodes in the network. The modified Dijkstra's algorithm presented here has a complexity of $O\left(N^{2}\right)$. The proof of correctness is omitted due to lack of space. Very briefly, the algorithm maintains two sets, i.e., $U$ and Q. $U$ contains the nodes that have not yet been visited, whereas $Q=V[G] \backslash U$. Temporary estimates of the max-RRT of all the paths originating at $s$ and ending at every vertex are maintained in $t$. The corresponding predecessors are maintained in prev. At the initial stage of the algorithm, all the vertices except the source are considered unreachable. At the beginning of every iteration of the while loop, the unvisited vertex with the largest RRT estimate (denoted by $t$ ) $u$ is extracted from $U$ and added to $Q$. If $u$ happens to be the destination (vertex $d$ ), then the max-RRT path has been found. Otherwise, we relax the adjacent vertices ${ }^{16}$ by examining whether the estimated RRT of a vertex can be improved if the path connecting $s$ and $d$ passes

\footnotetext{
${ }^{16}$ That is, node $u$ 's neighbors.
} 


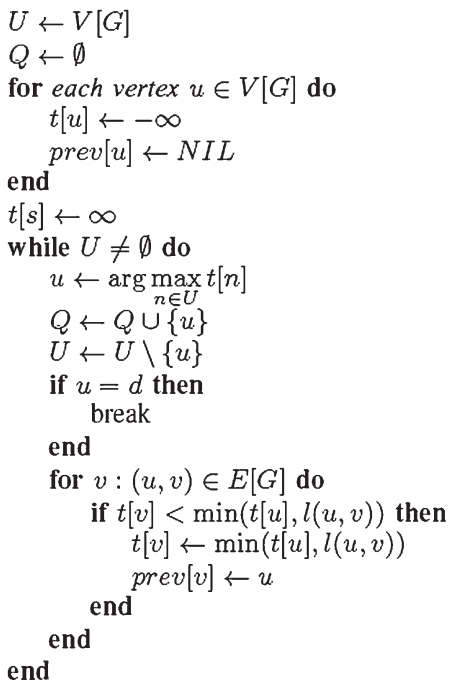

Fig. 6. Modified Dijkstra's algorithm for max-RRT path.

through $u$. If this is the case, then the max-RRT estimate is updated, and $u$ is set to be the predecessor node.

An additional feature that can exploit the redundancy of information resulting from the nature of LRT estimation can be included: two nodes sharing a wireless link will not necessarily have the same LRT estimate for the link in question. Because each of the nodes has sampled the channel at different time points, the estimates, which are based on the time series formed by the received packets, may differ. For this reason, we examine three different strategies as far as the definition of the nominal LRT of a specific link is concerned: 1) pessimistic scheme, where the smallest value of the two estimates is used; 2) neutral scheme, where the mean value of the two estimates is used; and 3) optimistic scheme, where the greatest value of the two estimates is used. Employing one of these schemes on the protocol level implies that a node needs to be aware of the neighbor's estimate for the link they share. This information can easily be included on the route construction or table update packets that are part typical routing protocols for ad hoc networks.

On a different note, LRT estimation is an online procedure; as has been mentioned, the initial estimates are based on a very few samples and are thus generally prone to being less accurate. To address this, we introduce a convergence criterion to classify the estimates. ${ }^{17}$ The nodes multiply all the LRT estimates produced before convergence has been achieved with a constant $c_{t}$, which is smaller than 1 ; the closer this constant is to 1 , the bigger trust we place on preconvergence estimates.

\section{A. Technical Details}

To evaluate the performance of the proposed estimation method when used for route construction, in conjunction with the proposed strategies, we have developed a simulation platform in C++ and Matlab, where we can compare the lifetime of the routes chosen by five different strategies: 1) minimum hop, where the route chosen is the shortest path between the

\footnotetext{
${ }^{17}$ More specifically, three successive LRT estimates should not differ more than three times the time interval between the time points on which those were made.
}

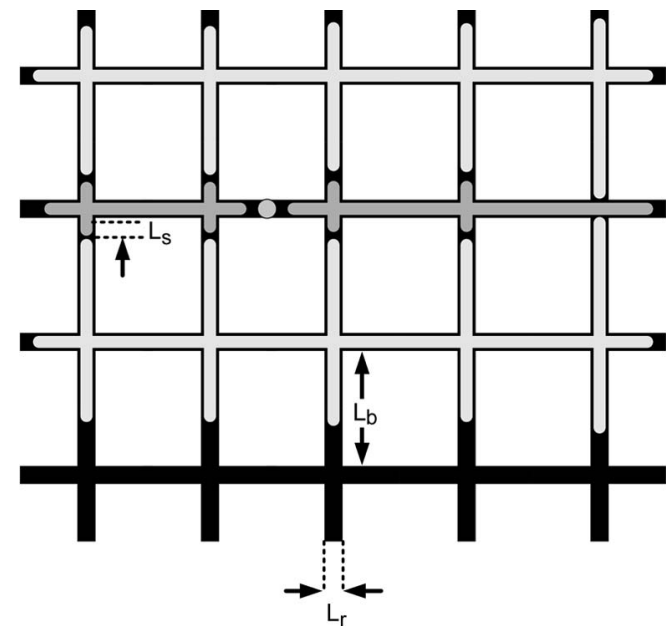

Fig. 7. Model of the grid on which the nodes are moving.

source and the destination; 2) SSNR based, where the metric used is the exponentially smoothed value of the SNR, i.e., SSNR, ${ }^{18}$ monitored on every link; 3) O-LRT, using the LRT estimates and the optimistic strategy; 4) N-LRT, using the LRT estimates and the neutral strategy; and 5) P-LRT, using the LRT estimates and the pessimistic strategy. The nodes are placed on a $L \times L$ square meter grid, with $L_{r}$ meter wide horizontal and vertical roads located at $L_{b}$ meter intervals (i.e., the grid consists of $M L_{b} \times L_{b} \mathrm{~m}^{2}$ building blocks), as depicted in Fig. 7. We assume this area to be closed in the sense that any nods leaving the area from the upper (left) side reenters it on the lower (right) side with the same speed and direction and vice versa. In addition, the distance between two nodes following the trajectories $\left(x_{a}(t), y_{a}(t)\right)$ and $\left(x_{b}(t), y_{b}(t)\right)$ is given by $\sqrt{x_{d}^{2}+y_{d}^{2}}$, where $x_{d}=\min \left(\left|x_{a}(t)-x_{b}(t)\right|, \mid L-\right.$ $\left.\left|x_{a}(t)-x_{b}(t)\right|\right)$, and $y_{d}=\min \left(\left|y_{a}(t)-y_{b}(t)\right|,|L-| y_{a}(t)-\right.$ $\left.y_{b}(t) \mid\right)$. This torus-resembling model of space has been used in [29]. ${ }^{19}$ At the beginning of each Monte Carlo iteration, the nodes are placed randomly on the grid, and mobile nodes are given a random direction and speed, where the latter is chosen from a uniform distribution between 2 and $10 \mathrm{~m} / \mathrm{s}$. The received power at the reference distance is taken to be equal to $P_{0}=-17 \mathrm{dBm}$, whereas the noise level is considered to be at $N_{0}=-95 \mathrm{dBm}$, and the threshold for efficient communication is set at $\mathrm{SNR}_{\mathrm{th}}=5 \mathrm{~dB}$. Similar as before, we are assuming that any packet above $0 \mathrm{~dB}$ can be detected. The packets are sent at random time points, with a rate of $\lambda=0.1$, and the SNR value is aggregated every $250 \mathrm{~ms}$. The path loss exponent $\gamma$ is taken to be equal to 3.5 and considered known to the method. Depending on the relative location of the nodes on the grid at any specific time point, a link is considered to be of good or bad quality. Nodes moving on the same road segment or that are close to road junctions ${ }^{20}$ are considered to be sharing a good quality link. Fig. 7 shows the good and bad link areas

\footnotetext{
${ }^{18} \mathrm{SSNR}=\alpha$ SSNR $+(1-\alpha)$ SNR, where we take $\alpha=0.15$. The route chosen maximizes the minimum SSNR over the links constituting the routing path.

${ }^{19}$ Note that there appears to be a typing error in the corresponding formula in the original paper.

${ }^{20}$ Nodes that are not farther away than $L_{s}$ meters from the closer junctions of their neighbor.
} 
TABLE II

PARAMETERS USED IN INDicATIVE Simulations

\begin{tabular}{cccccc}
\hline Scenario & $L_{S}(\mathrm{~m})$ & $L_{B}(\mathrm{~m})$ & $L_{R}(\mathrm{~m})$ & $N$ & $L(\mathrm{~m})$ \\
\hline 1 & 2.5 & 50 & 10 & 60 & 600 \\
2 & 2.5 & 50 & 10 & 40 & 600 \\
3 & 5 & 100 & 20 & 60 & 720 \\
4 & 2.5 & 90 & 10 & 40 & 600 \\
\hline
\end{tabular}

TABLE III

Mean Value of Lifetime ( $($ )

\begin{tabular}{rrcccc}
\hline Scenario & $P-L R T$ & $O-L R T$ & $N-L R T$ & Min. Hop & $S S N R$ \\
\hline 1 & 14.44 & 12.27 & 12.38 & 10.65 & 7.97 \\
2 & 10.93 & 10.99 & 10.92 & 8.60 & 7.28 \\
3 & 7.39 & 7.91 & 7.91 & 6.63 & 5.94 \\
4 & 14.00 & 14.02 & 14.13 & 12.90 & 10.42 \\
\hline
\end{tabular}

for a node in darker and lighter shades of gray, respectively. The standard deviation of shadow fading is taken to be equal to $\sigma_{1}=2 \mathrm{~dB}$ for nodes sharing a good quality link and $\sigma_{2}=4 \mathrm{~dB}$ otherwise, whereas the correlation distance is taken to be equal to $5 \mathrm{~m}$. Every Monte Carlo iteration lasts for $750 \mathrm{~s}$. During the course of each iteration, 50 route requests are made, and routes are chosen based on the five different algorithms. The source and destination nodes are chosen randomly. The routes are then monitored ${ }^{21}$ and their lifetimes ${ }^{22}$ are recorded.

\section{B. Evaluation}

For the sake of analyzing the behavior of the different route construction strategies with respect to the lifetime of the resulting routes, we run indicative simulations based on different scenarios for different values of the grid's parameters $\left(L_{S}, L_{B}\right.$, $L_{R}$, and $L$ ) and the number of nodes, which is denoted by $N$, as shown in Table II. For each scenario, five different Monte Carlo simulations are ran. The mean lifetimes of the routes chosen for the different scenarios are shown in Table III. We observe that pessimistic LRT performs better or close to other LRT strategies for all scenarios examined. The reason for this is that, particularly before convergence ${ }^{23}$ the estimation error distributions are left skewed (larger positive values are much more likely than negative ones), and therefore, the estimation methods tend to exaggerate the values of LRT. We can also see that all the LRT methods lead to routes of higher lifetime when compared with minimum hop or smoothed SNR.

\section{CONCLUSion AND Future Work}

In this paper, we have presented a method for estimating a link's residual time LRT. The method proposed is capable of taking the inherent nonlinearities of the temporal dependence of link quality into account and most importantly captures the effect of the minimum distance that will be achieved between two vehicles on the course of their movement on the link duration. In addition, it can produce LRT estimates regardless of whether

\footnotetext{
${ }^{21}$ We only monitor the cases where there was more than one possible route between the source and the destination, which accounts for the vast majority of the cases, as the nodes tend to form connected clusters.

${ }^{22}$ The time lapsed from the point of the route request until the point of the first link breakage among the links that constitute the route.

${ }^{23}$ As discussed, proconvergence estimates are taken into account after being multiplied with the trust constant $c_{t}$, which is taken to be equal to 0.5 .
}

the SNR of the link is improving or deteriorating over time. We have shown that the LRT estimates can successfully be used to identify the link with the longest LRT among two individual links, outperforming existing affinity-based methods, under different channel conditions. The expected value of the lifetime of the identified links based on the LRT estimates has been found to achieve values above or close to $93 \%$ of the expected value of the actual maximum lifetimes, whereas the same metric may reach values down to $69 \%$ for affinity-based schemes. Finally, we have demonstrated that the proposed estimation method can be beneficial for identifying long-lived routes, leading to the construction of routes that last much longer when compared with routes constructed based on the smoothed SNR metric.

A fruitful direction for the continuation of this paper would be to enhance the accuracy of the estimation methods by combining them with the use of Doppler shift measurements, knowledge of the nodes' location, and velocity vector and/or navigational information. The challenge would be to increase the method's accuracy, particularly under the presence of shadow fading, and during the first few estimations, when the link quality samples are very few. To this end, assuming knowledge of the general channel characteristics, such as the level of shadow fading, would also be beneficial. In the context of this paper, we have assumed that the nodes' speeds will not significantly change for the duration of the link. To facilitate adaptive parameter estimation, the mechanism described in [19] can be used together with the model proposed here to ensure that stale data are not used for the estimations. Newer estimates can then be used for route reconstructions when necessary. In addition, the predictions could be complemented with traffic statistics (e.g., probability for change of direction) for the road on which the vehicle is currently travelling or on knowledge of the route that the vehicle is going to follow. The objective of the route construction in this study was to maximize route lifetime, solely with respect to the RRT, to accurately capture the gain achieved under the proposed methods. A mechanism balancing the tradeoff between hop count and route lifetime, such as that described in [4], would benefit network performance even further. We also expect that constraining the number of hops during the route construction process would yield even longer lifetimes for LRTbased routing as the accuracy in the $R R T$ estimates deteriorates with the length of the route. Finally, the LRT estimations may be utilized to increase the robustness of other network-related functions, such as establishment of stable clusters, scheduling decisions, or quality-of-service-related provisioning.

\section{REFERENCES}

[1] J. Blum, A. Eskandarian, and L. Hoffman, "Challenges of intervehicle ad hoc networks," IEEE Trans. Intell. Transp. Syst., vol. 5, no. 4, pp. 347351, Dec. 2004.

[2] W. Su, S. J. Lee, and M. Gerla, "Mobility prediction and routing in ad hoc wireless networks," Int. J. Netw. Manage., vol. 11, no. 1, pp. 3-30, Jan./Feb. 2001.

[3] K. W. Chin, J. Judge, A. Williams, and R. Kermode, "Implementation experience with MANET routing protocols," SIGCOMM Comput. Commun. Rev., vol. 32, no. 5, pp. 49-59, Nov. 2002.

[4] Z. Cheng and W. B. Heinzelman, "Discovering long lifetime routes in mobile ad hoc networks," Ad Hoc Netw., vol. 6, no. 5, pp. 661-674, Jul. 2008.

[5] I. Chlamtac, M. Conti, and J. J. N. Liu, "Mobile ad hoc networking: Imperatives and challenges," Ad Hoc Netw., vol. 1, no. 1, pp. 13-64, 2003. 
[6] D. S. J. De Couto, D. Aguayo, B. A. Chambers, and R. Morris, "Performance of multihop wireless networks: Shortest path is not enough," SIGCOMM Comput. Commun. Rev., vol. 33, no. 1, pp. 83-88, Jan. 2003.

[7] H. Menouar, M. Lenardi, and F. Filali, "Improving proactive routing in VANETs with the MOPR movement prediction framework," in Proc. 7th ITST, Sophia Antipolis, France, 2007, pp. 1-6.

[8] J. Tang, G. Xue, and W. Zhang, "Reliable ad hoc routing based on mobility prediction," J. Combinatorial Optim., vol. 11, pp. 71-85, Feb. 2006.

[9] Z. J. Haas and E. Y. Hua, "Residual link lifetime prediction with limited information input in mobile ad hoc networks," in Proc. IEEE INFOCOM, 2008, pp. 1867-1875.

[10] Y. C. Tseng, Y. F. Li, and Y. C. Chang, "On route lifetime in multihop mobile ad hoc networks," IEEE Trans. Mobile Comput., vol. 2, no. 4, pp. 366-376, Oct.-Dec. 2003.

[11] S. Jiang, D. He, and J. Rao, "A prediction-based link availability estimation for mobile ad hoc networks," in Proc. IEEE INFOCOM, 2001, pp. 1745-1752.

[12] R. Dube, C. D. Rais, K. Y. Wang, and S. K. Tripathi, "Signal stability based adaptive routing for ad-hoc mobile networks," IEEE Pers. Commun., vol. 4, no. 1, pp. 36-45, Feb. 1997.

[13] C. K. Toh, "Associativity-based routing for ad-hoc mobile networks," Wirel. Pers. Commun., vol. 4, no. 2, pp. 103-139, Mar. 1997.

[14] H. M. Tsai, N. Wisitpongphan, and O. Tonguz, "Link-quality aware ad hoc on-demand distance vector routing protocol," in Proc. 1st Int. Symp. Wirel. Pervasive Comput., Jan. 2006, pp. 1-6.

[15] J. Singh, N. Bambos, B. Srinivasan, D. Clawin, and Y. Yan, "Proposal and demonstration of link connectivity assessment based enhancements to routing in mobile ad-hoc networks," in Proc. IEEE 58th Veh. Technol. Conf., 2003, vol. 5, pp. 2834-2838.

[16] S. Agarwal, A. Ahuja, J. P. Singh, and R. Shorey, "Route-lifetime assessment based routing (RABR) protocol for mobile ad-hoc networks," in Proc. IEEE ICC, 2000, vol. 3, pp. 1697-1701.

[17] R. S. Chang and S. J. Leu, "Long-lived path routing with received signal strength for ad hoc networks," in Proc. 1st Int. Symp. Wirel. Pervasive Comput., Jan. 2006, pp. 1-6.

[18] N. Sofra and K. K. Leung, "Estimation of link quality and residual time in vehicular ad hoc networks," in Proc. IEEE WCNC, Las Vegas, NV, 2008, pp. 2444-2449.

[19] N. Sofra and K. K. Leung, "Link classification and residual time estimation through adaptive modeling for VANETs," in Proc. IEEE 69th VTC Spring, Barcelona, Spain, Apr. 2009, pp. 1-5.

[20] N. Sofra, A. Gkelias, and K. K. Leung, "Link residual-time estimation for VANET cross-layer design," in Proc. IEEE IWCLD, Mallorca, Spain, 2009, pp. 1-5.

[21] M. Schwartz, Mobile Wireless Communications. Cambridge, U.K.: Cambridge Univ. Press, 2005.

[22] M. Gans, "A power-spectral theory of propagation in the mobile-radio environment," IEEE Trans. Veh. Technol., vol. VT-21, no. 1, pp. 27-38, Feb. 1972

[23] C. D. Iskanderp, "A matlab-based object-oriented approach to multipath fading channel simulation," Mathworks, Natick, MA, Tech. Rep. 18869, Feb. 2008.

[24] F. P. Fontán and P. M. Espineira, Modeling the Wireless Propagation Channel, 1st ed. Hoboken, NJ: Wiley, 2008, ch. 3, pp. 66-71.
[25] T. Rappaport, Wireless Communications: Principles and Practice. Upper Saddle River, NJ: Prentice-Hall, 2001.

[26] C. E. Perkins and E. M. Royer, "Ad-hoc on-demand distance vector routing," in Proc. 2nd IEEE WMCSA, Washington, DC, 1999, p. 90.

[27] C. E. Perkins and P. Bhagwat, "Highly dynamic destination-sequenced distance-vector routing (DSDV) for mobile computers," SIGCOMM Comput. Commun. Rev., vol. 24, no. 4, pp. 234-244, 1994.

[28] T. H. Cormen, C. E. Leiserson, R. L. Rivest, and C. Stein, Introduction to Algorithms, 2nd ed. Cambridge, MA: MIT Press, 2001.

[29] Z. J. Haas, "A new routing protocol for the reconfigurable wireless networks," in Proc. IEEE Int. Conf. Univ. Pers. Commun., 1997, pp. 562-566.

Nikoletta Sofra (M'11) received the Diploma in electrical and computer engineering from the National Technical University of Athens, Athens, Greece, in 2006 and the Ph.D. degree from Imperial College London, University of London, U.K., in 2010.

She has worked with the Mobile Environmental System Across Grid Environments (MESSAGE) and International Technology Alliance (ITA) projects In the Summer of 2005, she was with Siemens AG, Munich, Germany. In 2009, she was an Intern with the Watson Research Center, IBM, Hawthorne, NY. She is currently with Audi Electronic Venture GmbH, Audi AG.

Athanasios Gkelias (M'10) received the Diploma in electrical and computer engineering from Aristotle University of Thessaloniki, Thessaloniki, Greece, in 2000 and the M.Sc. and Ph.D. degrees in telecommunications from King's College London, London, U.K., in 2001 and 2005, respectively.

In the past, he was a Visiting Researcher with Bell-Labs Research Centre, Alcatel-Lucent, U.K., as well as the Athens Information Technology, Athens, Greece. Since 2006, he has been a Postdoctoral Researcher with Imperial College London. He has published several technical journal and conference papers and has been a Technical Program Committee member of various conferences. His research interests lie in the area of wireless ad hoc, sensor, and mesh networks, particularly in the performance analysis and design of medium access control and quality-of-service routing protocols and cross-layer issues.

Kin K. Leung (F'01) received the B.S. degree from the Chinese University of Hong Kong, Sha Tin, Hong Kong, in 1980 and the M.S. and Ph.D. degrees from the University of California at Los Angeles in 1982 and 1985, respectively.

He joined AT\&T Bell Labs in 1986 and worked at its successor companies, AT\&T Labs and Bell Labs of Lucent Technologies, until 2004. Since then, he has been the Tanaka Chair Professor in Electrical and Electronic Engineering (EEE) and Computing Departments, Imperial College London, London, U.K. He serves as the Head of Communications and Signal Processing Group and as the Deputy Director for the University Defense Research Center in Signal Processing with the EEE Department, Imperial College.

Dr. Leung is a member of the IEEE Fellow Evaluation Committee for Communications Society (2009 to 2011). He received the Distinguished Member of Technical Staff Award from AT\&T Bell Labs in 1994 and was a corecipient of the 1997 Lanchester Prize Honorable Mention Award. He received the Royal Society Wolfson Research Merits Award from 2004 to 2009. 